\title{
EXPERIENCIAS DIDÁCTICAS PARA EL MEJORAMIENTO DE LA PRÁCTICA PEDAGÓGICA DEL PROFESOR DE ARTES PLÁSTICAS
}

\author{
Julieta Castro Bonilla \\ Profesora de la Escuela de Formación Docente \\ Investigadora del Instituto de Investigación en Educación (INIE) \\ La enseñanza de la educación artística desarrolla \\ la iniciativa y la creatividad, estimula la imaginación, \\ fomenta el orgullo por la destreza, desarrolla la capacidad de \\ planificación y, en algunos campos artísticos, \\ ayuda a los estudiantes a cooperar.
}

Elliot W. Eisner.

\begin{abstract}
Resumen: El texto que a continuación presento, surge de una investigación en la que participan docentes que imparten lecciones en instituciones del sistema educativo y se preocupan por renovar sus prácticas pedagógicas con el propósito de que sus estudiantes ᄀ-niños y adolescentes-apliquen los aprendizajes mediante nuevas formas de expresión artística.

En el artículo se menciona la metodología que aplica esa investigación, la cual se organiza mediante "fases" que facilitan espacios reflexivos que permiten valorar el trabajo docente mediante el enfoque cualitativo que promueve la investigación-acción en el aula.

También se hace referencia a la fundamentación de una práctica pedagógica que le permite al profesor y a sus estudiantes, la posibilidad de asignar nuevos significados al trabajo que se realiza dentro y fuera del contexto escolar.

Finalmente, se ofrecen algunas propuestas metodológicas que se dirigen de manera especial a quienes imparten lecciones de artes plásticas en la educación secundaria, aunque se pueden adecuar a otras poblaciones estudiantiles, mediante la planificación de proyectos que tiendan a mejorar las experiencias artisticas de quienes participan en los procesos de aprendizaje. Se espera que el texto facilite la organización de investigaciones que favorezcan la innovación en el aula.
\end{abstract}

\section{Introducción}

El presente artículo se origina en la investigación La práctica pedagógica construida a partir de las creencias, concepciones y estilos de pensamiento del personal docente. Un espacio para su reflexión desde la expresión artística", en la que participé junto a otras profesoras de la Universidad de Costa Rica. La investigación se refiere a la reconstrucción de la práctica pedagógica de los docentes participantes para transformarla a partir de sus conocimientos, creencias, concepciones y modelos de pensamiento, e incorporar la expresión artística como un recurso didáctico que posibilita la adquisición de aprendizajes significativos por parte de los estudiantes. Para una mayor comprensión del documento que se presenta, se hace referencia a Marcelo (1987), quien señala que las concepciones y creencias sobre los procesos 
Palabras clave: Investigadoras, docentes que laboran en el sistema educativo, estudiantes, expresión artística, enseñanza de las artes plásticas, educación integral.

Abstract: The following text comes from the research of teachers that work in the institutions of the education system and are concerned about the renewal of their pedagogical practices. The purpose of this renewal is to give their students -children and teenagers- a way to apply their knowledge with new forms of artistic expression.

The methodology applied in this research is mentioned in the article. The methodology is divided into "phases" that facilitate spaces for thought; these spaces allow the appraisal of the teacher's work through the qualitative focus that encourages action research in the classroom.

This article also mentions the foundations of a pedagogical practice for the construction of new meanings for the work of students and teachers in and out of the school's context.

Finally, it offers some methodological proposals specially targeted towards fine arts teachers in secondary school. These proposals may also be adapted to other student populations. The latter can be achieved by planning projects about improving the artistic experiences of the participants of the learning processes. This text is expected to make the organization of research work for innovation inside the classroom easier.

Key words: Teachers, students, artistic expression, teaching of fine arts, integral education. de enseñanza y aprendizaje emanan de la experiencia y de la formación académica de quien ejerce la docencia. Una creencia personal no es sólo el producto de una norma colectiva, sino la síntesis de teorías propias que se ajustan poco a poco a las necesidades y requerimientos externos. La constante interacción con diferentes docentes, compañeros, amigos y especialistas en determinados campos del saber, llevan a la persona a la adquisición de conocimientos y aprendizajes que le permiten ser parte del núcleo social; por lo tanto, la construcción de esos conocimientos constituye un proceso mediante el cual, el sujeto desde sus propias prácticas, le asigna valores y significados a los estímulos que percibe, organiza la información que recibe del contexto, la interioriza para formar posteriormente, sus propias concepciones de acuerdo con al ambiente en que se desenvuelve. Las creencias o teorías implícitas se forman en el interior de cada persona, de manera consciente o inconsciente, son producto de la interacción con el medio y permiten hacer representaciones individuales basadas en prácticas sociales y culturales que intervienen de manera directa en el trabajo que desempeña el docente.

Como parte de los resultados de la misma investigación, se señala que los significados que un individuo concede a sus experiencias y conocimientos, forman parte del conjunto de teorías que va interiorizando e inciden de manera directa en su vida personal y profesional. De esta manera, cada docente elabora su conjunto de creencias a las que le asigna un lugar en su interior, les atribuye su propio significado, las modifica, las enriquece o las desecha conforme va adquiriendo experiencia de vida, lo expuesto se considera fundamental para la labor que desempeña el profesor como parte de su trabajo de aula, dado que las creencias o teorías implícitas, constituyen parte de su aprendizaje, y también son consideradas el producto y el resultado de la interacción con sus colegas y estudiantes, situación que fortalece y refuerza su visión de mundo. 
Para explicar el desarrollo de la práctica pedagógica que construyen los docentes a partir de sus creencias, concepciones y estilos de pensamiento; la investigación se inicia a partir de la observación del trabajo de aula que desarrollamos las responsables de la investigación. El deseo de participar en procesos de auto-evaluación sobre el trabajo que realizamos las investigadoras, facilitó la labor de observación de la práctica docente que realizaron los profesores participantes en la investigación.

Cabe destacar que el documento también se sustenta en mi trabajo como investigadora y profesora de artes plásticas para educación secundaria; en la lectura de textos relativos al tema, y finalmente, en mis vivencias académicas y profesionales como docente universitaria impartiendo cursos relacionados con las áreas de pedagogía, didáctica y artes plásticas, dirigidos a estudiantes de educación preescolar, primaria y secundaria, sin obviar mis propias creencias y teorías implícitas que también se constituyen en parte de mi aprendizaje.

En este artículo, el área de las artes plásticas, se considera el "eje medular" que permite un replanteamiento para redefinir una práctica pedagógica que facilita la aplicación creativa de conocimientos, habilidades y destrezas de parte de la población estudiantil. Para las responsables de la investigación, la formación académica que reciben los futuros educadores durante el transcurso de su carrera universitaria es considerada fundamental, ya que les permite ajustarse y adecuar las políticas curriculares (del Ministerio de Educación Pública y los lineamientos que solicitan las direcciones regionales y centros de enseñanza) a su trabajo de aula sin problema alguno. Según Rueda y Díaz (2000), la formación académica debe dar relevancia a los aspectos académicos y pedagógicos, para que tanto la persona que ejerce la docencia, como sus estudiantes, puedan mantener un constate y mutuo desarrollo personal y social.

El documento también hace referencia a la propuesta metodológica de la investigación mencionada, la cual se organiza mediante "fases" que permitieron valorar la práctica pedagógica mediante el enfoque cualitativo que promueve la investigación-acción en el aula. Las experiencias desarrolladas por los docentes en los diferentes centros educativos, favorecieron en sus estudiantes, el desarrollo de la creatividad, iniciativa, imaginación, así como la expresión de sus conocimientos y aprendizajes mediante la aplicación de variadas técnicas pictóricas.

Se espera que las experiencias de integración curricular que se describen al final del artículo, se conviertan en insumos para que puedan ser aplicadas por docentes de artes plásticas y otras personas interesadas en el tema, de manera que puedan propiciar espacios de reflexión sobre su práctica educativa, la valoren y puedan descubrir cuánto han aprendido y cuánto les falta por aprender.

\section{La formación humanística, artística y pedagógica de los docentes de artes plásticas participantes en la investigación}

La literatura estudiada señala que la acción docente se construye en el aula a partir de:

a) los conocimientos adquiridos durante los procesos de formación formal e informal, con énfasis en la educación superior recibida.

b) de las experiencias, creencias y concepciones personales que posee cada docente.

De acuerdo con lo señalado, la formación académica, artística y pedagógica se considera relevante para el desarrollo de los procesos de aprendizaje que el docente debe estimular como parte de su trabajo de aula. Por ese motivo, a continuación se hace referencia a la Universidad de Costa Rica como Institución formadora de los docentes que participaron en la investigación. 
La estructura curricular del Plan de "Bachillerato en la Enseñanza de la Artes Plásticas", se encuentra conformado por tres áreas consideradas fundamentales en la formación de estos docentes: área de Cultura General Humanística, área de Especialización Artística y área de Formación Pedagógica.

\section{- Área de cultura general humanística}

La visión humanista que posee el plan de estudios responde al planteamiento filosófico de la Universidad de Costa Rica, el cual consiste en inspirar y desarrollar el interés permanente por la cultura general y humanística, así como crear una conciencia crítica por la problemática costarricense, latinoamericana y mundial. (Universidad de Costa Rica. Consejo Universitario, Estatuto Orgánico, 2000)

Acorde con esa visión de humanismo, esta área la conforman los siguientes cursos: Humanidades, actividad deportiva, artística, repertorios, Seminarios de Realidad Nacional I y II, así como el Trabajo Comunal Universitario de 300 horas de trabajo debidamente planificado y sistematizado que el estudiante debe brindar al país sin remuneración alguna.

La fundamentación filosófica que ofrece la Escuela de Estudios Generales de la Universidad de Costa Rica se concreta mediante la formación humanista que le permite al futuro educador participar en la educación desde una perspectiva holística.

\section{- Área de especialización artística}

Corresponde a la formación artística impartida por la Escuela de Artes Plásticas. De acuerdo con el planteamiento curricular que ofrece esta Escuela, el Plan de estudios ofrece a los estudiantes una formación general mediante los siguientes:
Dibujo, Diseño, Introducción a la Pintura, Introducción a la Escultura, Historia del Arte, Filosofía del Arte, Cerámica, Serigrafía, Xilografía, Grabado en Metal, Textiles. Además de los cursos mencionados, los estudiantes deben aprobar otros que se denominan "énfasis", los cuales le permiten profundizar su área de formación artística en los siguientes énfasis que ofrece la Escuela: pintura, escultura, cerámica, grabado y artes gráficas.

Los cursos que corresponden a esta área expresiva le permiten al docente una formación artística que los faculta para que puedan planificar enriquecedoras experiencias de arte, con el propósito de que sus estudiantes pueden alcanzar los objetivos del programa de artes plásticas que propone el Ministerio de Educación Pública y los que pueda formular el mismo orientador del aprendizaje.

\section{- Área de formación pedagógica}

Corresponde al conjunto de cursos que imparten la Escuelas de Formación Docente en coordinación con las de Administración Educativa y la Escuela de Orientación y Educación Especial.

Esta área de formación pedagógica comprende dos sub-áreas: los Fundamentos educativos y la Didáctica.

- Los Fundamentos Educativos estudian las corrientes filosóficas, sociológicas y psicológicas de la educación general, aunque se enfatiza en la educación costarricense.

- La Didáctica, incluye el estudio de conocimientos y destrezas necesarias para desarrollar los procesos de enseñanza y aprendizaje. Esos cursos tienen como referencia inmediata y campo de aplicación, el trabajo docente en las aulas del tercer ciclo de educación diversificada. Esa subárea se completa con el estudio de 
aspectos básicos de la investigación educativa orientados a la elaboración de pequeñas investigaciones. Plan de Profesorado y Bachillerato en la Enseñanza. 1991.

El propósito del área de formación pedagógica es estimular la actitud hacia la docencia, así como desarrollar los conocimientos, habilidades y las destrezas necesarias para que el futuro docente sea capaz de permitirle al joven estudiante, un desarrollo armónico de su personalidad, en donde las actividades que organiza dentro del contexto de aula estén encaminadas a facilitar procesos de construcción y de apropiación del conocimiento, así como al desarrollo de las habilidades y destrezas sugeridas en el programa de Artes Plásticas del Ministerio de Educación Pública.

Para la autora del artículo, la formación académica recibida por quienes concluyen el programa de estudios: Bachillerato en la Enseñanza de la Artes Plásticas (y que participaron en la investigación), es idónea, ya que los conocimientos y aprendizajes adquiridos en las áreas artística, pedagógica y humanística, favorecieron la aplicación de nuevas formas de enseñanza y en consecuencia la adquisición de nuevos aprendizajes en sus jóvenes estudiantes.

\section{Algunas reflexiones sobre la práctica pedagógica}

Respecto de este tema, Fierro, Fortoul y Rosas (2002, p. 21), señalan: ....se entiende por práctica pedagógica la praxis social, objetiva e intencional en la que intervienen los significados, las percepciones y las acciones de los agentes implicados en el proceso: maestros, alumnos, autoridades educativas y padres de familia, así como los aspectos político-institucionales, administrativos $\mathrm{y}$ normativos, que según el proyecto educativo de cada país delimitan la función del maestro. El concepto de práctica pedagógica a la que se hace referencia, aunada a la experiencia profesional de quienes participamos en la investigación, ponen en evidencia al docente como la persona responsable de planificar procesos de comunicación que ofrezcan nuevos significados al trabajo que desarrollan sus estudiantes.

La teoría estudiada señala que la práctica pedagógica implica un marco de acción que lo constituyen los siguientes elementos: los procesos de formación académica, la experiencia profesional, las creencias, las concepciones pedagógicas, las políticas curriculares, la visión de mundo y la forma de ser de quien organiza y conduce el trabajo de aula; los elementos señalados se deben relacionar de manera directa con el contexto sociocultural, ya que el hecho educativo no es aislado y se ve influenciado por factores externos e internos considerados inherentes al trabajo que desempeña el educador.

En el párrafo anterior se evidencia que le corresponde al docente asumir un papel protagónico en el desarrollo de los procesos de enseñanza y aprendizaje, así como poseer la capacidad para modificar el currículo escolar al contexto en que desarrolla su trabajo. Lo expuesto concuerda con Gimeno (1993, p. 196), al señalar que... "el curriculum moldea a los docentes, y éste es traducido en la práctica por ellos mismos".

Al referirme a la práctica pedagógica, se considera necesario hacer alusión a los conceptos de enseñanza y aprendizaje que Flores (1999), define como dos procesos correlativos, inseparables el uno del otro, relacionados como causa y efecto, aunque sabemos que muchos de los aprendizajes se obtienen de la vida, sin que medie ningún tipo de enseñanza. Como procesos correlativos, la enseñanza y el aprendizaje no se pueden confundir el uno con el otro. Según el mismo autor, el aprendizaje real ocurre en el interior de cada persona que aprende, es subjetivo y su dominio se pueda exteriorizar mediante acciones específicas como son las palabras y las diversas formas creativas. La enseñanza es una actitud intersubjetiva y se considera el producto de una 
interacción que se lleva a cabo entre varios sujetos -al menos dos- sobre algún tema determinado o material previamente seleccionado por el docente para suscitar una conversación, reflexión o acción compartida y de la que se espera algún aprendizaje. Rueda y Díaz (2000, p. 261), conciben la enseñanza como un acto de comunicación donde el profesor es el responsable de adaptar y de otorgar significados al currículum y de poner a los estudiantes en contacto con los contenidos mediante la creación de un ambiente adecuado para enseñar y aprender; así como de permitirle a sus estudiantes la concreción de sus conocimientos $\mathrm{y}$ aprendizajes en forma escrita, oral y mediante diversas formas de expresión.

Según Flores (1999), la enseñanza de calidad requiere de un profesor que tenga claridad acerca de lo que va a enseñar, por lo que de manera sistemática debe hacerse los siguientes cuestionamientos: ¿Qué tipo de ser humano quiere formar? ¿Con qué experiencias crece y se desarrolla? ¿Quién debe impulsar el proceso educativo? ¿Con qué métodos y técnicas puede alcanzar aprendizajes significativos?, estas preguntas deben ser desarrolladas de manera amplia por especialistas en el tema; sin embargo, como parte de su práctica pedagógica, el docente debe tener la capacidad para abordarlas de forma transdisciplinaria, por lo que requiere de una excelente preparación académica, pedagógica y experiencia; además de sentir gusto, orgullo y satisfacción por lo que hace. Lo expuesto le permite al docente, no solo reflexionar en "cuanto al objeto de estudio o del conocimiento", sino comprender que como planificador de actividades, no debe transmitir ni aplicar soluciones que otros proponen, sino que debe crear su propia práctica pedagógica teniendo presente las necesidades de sus estudiantes y el contexto de aula. Para que lo expuesto se pueda llevar a la práctica, se reitera la necesidad de que los educadores posean una preparación académica idónea, que les permita enlazar de manera creativa e innovadora, los diversos componentes del currículo escolar con las actividades que se realizan dentro y fuera de las aulas. Si lo que se enseña no tiene relación con el contexto en que se desarrolla la labor educativa, la relación entre teoría y práctica es incoherente y se podrían obtener resultados inesperados.

Para que lo expuesto se pueda concretar, le corresponde al profesor la tarea de planificar técnicas metodológicas que permitan el logro de los objetivos curriculares tomando en cuenta la capacidad intelectual, las formas de aprender, los intereses y dificultades, el nivel académico y económico, así como el contexto escolar de sus estudiantes, sin obviar los intereses y experiencias que surgen a partir de sus propias concepciones y creencias personales, las cuales forman parte del conjunto de teorías que va interiorizando e inciden de manera directa en su vida personal y profesional.

Alvarado y Murano (1990), consideran que se deben organizar experiencias de artes plásticas ya que le permiten al docente:

- Trabajar con afecto y de manera empática con sus estudiantes.

- Respetar por igual todas las ideas procedentes de sus estudiantes, así como disfrutar con ellos de sus descubrimientos.

- Favorecer el proceso más que el resultado, ayudándole a cada uno a autoevaluarse.

- Sensibilizar al alumno frente a la naturaleza y frente a su condición humana.

- Atender la problemática de cada uno en particular, pero sin perder de vista al grupo.

- Promover temas y asuntos de interés.

- Promover cantidad y variedad de materiales y técnicas para que puedan expresar y representar lo que deseen.

- Tener presente que para fomentar cambios de actitud en los alumnos, debemos empezar por cambiar nosotros.

Las actitudes mencionadas, también fomentan el desarrollo del pensamiento 
divergente, lateral o creativo, en el que no existe respuesta única a un problema, como es el caso de la matemática o física. Recordemos que en el área de la expresión de las artes se pueden ofrecer tantas respuestas como educandos.

La cita anterior pone de manifiesto que el docente debe planificar su práctica pedagógica, recordando que en todo proceso de enseñanza y aprendizaje se evidencian cuatro elementos que merecen especial atención: el estudiante, el docente, el conocimiento y el desarrollo de destrezas. La enseñanza como práctica social y por formar parte de la educación, se debe organizar tomando en cuenta no solo los elementos mencionados, sino la planificación de unidades didácticas en las que se deben tener presentes la identificación de los objetivos, contenidos, actividades, recursos y evaluación. Cabe señalar que -en el trabajo de investigación realizado-: los objetivos, los temas seleccionados, así como las destrezas y habilidades se entrelazaron a otras áreas del currículo escolar de manera coherente, flexible y creativa, lo cual dio como resultado el logro de aprendizajes que impactaron de manera significativa en la población estudiantil.

\section{Procedimiento metodológico de la investigación}

En este apartado se hace referencia al procedimiento metodológico de la investigación señalada al inicio del artículo, el cual facilitó la planificación y aplicación de propuestas didácticas con excelentes resultados. El proyecto investigativo se desarrolló a partir de fases que implicaron espacios de reflexión, seguimiento y valoración de la práctica pedagógica de acuerdo con el enfoque cualitativo que promueve la investigación-acción en el aula. Las fases expuestas a continuación, se desarrollan sobre "eventos pedagógicos", entendidos éstos como los actos pedagógicos que realizan los educadores con sus estudiantes como parte fundamental de la vida cotidiana del centro educativo. Se pretende que la metodología aplicada le permita a otros educadores la posibilidad de generar conocimiento a partir de sus propias prácticas educativas, las cuales favorecen el desarrollo humano, Romero (1998).

\subsection{Fases de la investigación}

Fase $N^{o}$ 1: Reflexión sobre la práctica pedagógica de las investigadoras

Según Romero (1998, p. 92):

...las fases y etapas son períodos y sub-períodos en el desarrollo de los fenómenos. El desarrollo es la forma de existencia real de los fenómenos del mundo en un permanente proceso. Es su capacidad interna de auto movimiento, de auto dinamización de su devenir; de estar siendo y dejando de ser progresivamente o involutivamente. Cuando el ser epistémico aborda un campo de investigación se enfrenta al estar siendo y dejando de ser; es decir: al proceso.

Esta primera fase se desarrolla sobre los actos pedagógicos que realizaron las investigadoras como parte de su trabajo de aula, es decir, con sus estudiantes universitarios.

La Fase $\mathbf{N}^{\mathbf{0}} \mathbf{1}$ corresponde al primer acercamiento a la práctica pedagógica que las investigadoras desarrollamos en los cursos que impartimos ${ }^{1}$; por lo que se elaboró una guía de observación que permitió detectar los procesos de enseñanza que se refieren a las actividades metodológicas aplicadas. Se indagó entre las investigadoras, hasta dónde nuestras creencias, concepciones, estilos de pensamiento, nuestra formación académica y experiencia, incidían en el trabajo que como profesoras desarrollamos; por lo que se procedió a la deconstrucción ${ }^{2}$, al análisis y a la valoración de nuestra práctica pedagógica. La información que se obtiene (mediante la guía de observación), al concluir el período de reflexión de la práctica pedagógica de las investigadoras, se utiliza para desarrollar procesos de discusión y reflexión. Cada investigadora fue observada por las otras colegas en el transcurso de un mes. La sistematización de la 
experiencia se organiza de acuerdo con las siguientes categorías, las cuales responden al cuestionario preparado para tal efecto:

- Diagnóstico sobre las concepciones, creencias, percepciones y estilo docente de cada una de las investigadoras.

- $\quad$ Planificación de la práctica pedagógica.

- $\quad$ Estrategias didácticas que propician la integración de la expresión artística por parte los estudiantes que conforman los cursos.

- Reflexión de la práctica pedagógica que desarrollan las personas participantes en relación con la integración de la expresión artística.

- Lecciones aprendidas con la experiencia.

\section{Fase $N^{\circ}$ 2: Selección de las personas participantes}

El grupo lo conforman 18 docentes provenientes de las carreras de: educación preescolar, primaria, y educación secundaria (con énfasis en la enseñanza de la filosofía, música y artes plásticas), que laboran en instituciones públicas y privadas, en zonas rurales y urbanas. El criterio de selección se apoya en el interés y disponibilidad por conocer, analizar y reflexionar sobre su práctica pedagógica a partir de la expresión artística. Se reitera que quienes integran el proyecto, poseen una formación académica sólida y una importante experiencia en el aula que los motiva a reflexionar y a revisar de manera constante su propia práctica educativa para transformarla.

\section{Fase $N^{o}$ 3: Autorreflexión de la práctica pedagógica de las personas participantes}

A partir de la sistematización de las experiencias desarrolladas en el trabajo de aula de las investigadoras y tomando en cuenta las categorías señaladas en la Fase $\mathrm{N}^{\mathrm{o}} 1$, se propone una metodología que promueve espacios de reflexión y autorreflexión de la práctica pedagógica de los docentes, a la que se incorporan las vivencias y experiencias personales y profesionales, así como el conocimiento que poseen de los contenidos que deben desarrollar como parte de su trabajo de aula. La metodología que se promueve está constituida por los siguientes lineamientos:

a. Elaboración de un instrumento que permitió hacer un diagnóstico sobre las creencias, concepciones y estilos de pensamiento de los docentes participantes en cuanto a la práctica pedagógica que desarrollan. Este instrumento favoreció escribir y reflexionar sobre los aspectos antes señalados.

b. Tomando en cuenta los resultados de ese diagnóstico se programaron talleres con los docentes participantes; se propiciaron espacios de reflexión que dieron como resultado procesos que facilitaron la deconstrucción de la práctica pedagógica. Se seleccionaron los contenidos del currículum que se podían asociar con otras áreas y finalmente se organizaron planteamientos integradores. En otras palabras, el concepto de integración curricular quedó manifiesto en esos talleres.

c. La reconstrucción de dicha práctica se realiza a partir de las siguientes actividades:

- Identificación de sus propias creencias, concepciones, estilos de pensamiento de los participantes. Posteriormente, se explicó la necesidad de conocer cómo conceptualmente cada participante podía expresar los aspectos señalados anteriormente (sus propias creencias, concepciones y estilos de pensamiento), como parte de su acción dentro del contexto de aula. 
- Contrastar si los referentes conceptuales indicados en el punto anterior, a veces explícitos, otras veces implícitos, eran considerados por cada participante en su práctica pedagógica.

- Analizar y reconocer el grado de comprensión que cada docente poseía sobre los contenidos curriculares, así como las actividades didácticas adecuadas para ser desarrolladas en el aula.

- Incluir la expresión plástica como un recurso metodológico que permite la vivencia de los contenidos seleccionados como parte del trabajo de aula.

- Acompañar a los docentes participantes en el proceso de reconstrucción mediante lecturas afines y actividades didácticas de interacción.

d. Los talleres fueron participativos y las actividades se organizaron a partir de las inquietudes, dudas e intereses planteados por los docentes participantes e investigadoras. Cada taller abarcó aspectos específicos que facilitaron los procesos de análisis y reflexión de la práctica pedagógica, así como la integración artística unida al currículum de la siguiente manera:

- los procesos de observación que realizaron las investigadoras al personal docente en los diferentes centros de enseñanza, permitieron la realimentación del trabajo que cada persona organiza como parte de la programación de actividades. Lo anterior llevó implícito la construcción de sus conocimientos y aprendizajes.

- para finalizar esta etapa se concretaron procesos de autoevaluación con la población docente participante. e. Las investigadoras retomaron los resultados obtenidos, y de manera conjunta con los docentes se plantearon nuevas estrategias para mejorar la práctica pedagógica. Se observó y se realimentó el desempeño de los docentes participantes en el contexto de aula. Se realizaron nuevos procesos de evaluación formativa con las personas involucradas, incluyendo la valoración de las investigadoras hacia el trabajo desarrollado.

Para concluir la Fase $\mathbf{N}^{\mathbf{o}} \mathbf{3}$, deseo destacar que el personal docente se dio a la tarea de reflexionar y valorar su práctica pedagógica de manera constante y sistemática, así como de organizar procesos de enseñanza que facilitaron la concreción de aprendizajes de parte de sus estudiantes. Durante el proceso de planificación, la población estudiantil también participó ofreciendo sugerencias y opiniones. En otras palabras, la organización de los planteamientos fueron ajustados a los temas, edades, recursos didácticos, sin obviar el contexto escolar. Algunas propuestas metodológicas de integración se describen en el apartado $\mathrm{N}^{\circ} 5$.

\section{Fase $N^{o}$ 4. Elaboración de un texto final}

Para concretar los resultados obtenidos de la investigación y comprender las relaciones entre la práctica pedagógica y la expresión artística como agente transformador del quehacer docente y su repercusión en los procesos de aprendizaje de sus estudiantes, el equipo de investigadoras prepara un libro de texto que sintetiza el proceso realizado.

\section{Propuestas metodológicas de integración organizadas por los participantes}

En este apartado se describen algunas de las experiencias metodológicas que 
los profesores de artes plásticas planificaron y llevaron a la práctica como resultado de los procesos de la investigación. Cabe señalar que en todo momento de destacó el concepto de "integración" entre las artes plásticas y otras áreas del currículo escolar. La teoría estudiada como parte de la investigación, aunada a la formación (académica y artística) y a la experiencia profesional de cada docente, le permitió conceptuar su práctica pedagógica a partir de sus propias creencias, a las que se les atribuyó el logro de nuevos aprendizajes. Como parte del proceso de planificación de las propuestas metodológicas estuvieron presentes las características personales de la población adolescente, su creatividad e iniciativa, los conocimientos previos, sus experiencias de arte y el contexto de aula en que se desarrolló el trabajo. Se espera que los comentarios y reflexiones que pueda generar en el lector el artículo que se presenta, le permita organizar investigaciones y experiencias didácticas que puedan contribuir a la transformación, al mejoramiento y al enriquecimiento de prácticas pedagógicas que generen interesantes aprendizajes.

Como se ha explicado a lo largo del documento, en la práctica pedagógica que desarrollara cada docente se evidenció su ıormaciun acauemica, sus propias creencias, $\mathrm{su}$ experiencia profesional, sus concepciones de vida, su estilo de pensamiento, esos aspectos favorecieron la promoción de actividades que implicaron novedosos y atractivos descubrimientos durante cada lección. Según Klingler y Vadillo (1997), no existe una teoría exclusiva y sustentable, y no hay fórmulas universales para realizar el trabajo de aula, por lo que cada situación en clase debió tratarse como una experiencia única que requirió de soluciones específicas dependiendo del contexto, de los niveles de desarrollo de los estudiantes, de los temas y de los objetivos curriculares. Los docentes que participaron en la investigación tomaron conciencia de que los métodos de enseñanza deben enfatizar el aprendizaje activo y centrado en el alumno, al considerar que una visión constructivista del conocimiento favorece el trabajo de aula. Es importante señalar que -en la medida de lo posible- los docentes con la colaboración de sus estudiantes, crearon dentro del aula ambientes que permitieron la exploración de diversas formas de trabajo, tanto en las actividades que presentaron una orientación teórica, como las prácticas o de taller.

Algunas de las propuestas metodológicas que planificaron y desarrollaron los docentes participantes se describen a continuación:

- La educación ambiental y las artes plásticas. Después de leer un texto relativo al área de educación ambiental, los estudiantes se formularon preguntas y respuestas entre ellos mismos. Esta experiencia, además de beneficiar los procesos de investigación y aclaración de dudas, favoreció la comunicación y el respeto hacia las ideas de sus compañeros. Posterior a este período, el docente los invitó a relacionar el tema estudiado con el área de las artes plásticas. La idea es que fueran ellos los mismos quienes articularan ambas áreas. Cuando los estudiantes establecieron las posibles relaciones, diseñaron dos murales con temas alusivos a la naturaleza los cuales fueron coloreados con tintes naturales producidos mediante extractos de flores, tallos, hojas, vegetales y tierra. Finalmente, los docentes que imparten enseñanza de las ciencias también expresaron sus opiniones y comentarios.

- Proyectos de investigación. Se invitó a los estudiantes a recordar temas de otras áreas del currículo escolar que estuvieran estudiando en ese momento. De manera individual, cada uno escogió el contenido de su agrado y realizó una pequeña reflexión en forma escrita, ya sea mediante una poesía, canción o adivinanza. Posteriormente, representaron 
gráficamente el tema seleccionado mediante la aplicación de materiales de desecho (papeles, corchos, cartones, conchas, entre otros). Para concluir esta etapa, los estudiantes, además de referirse al tema que motivó su trabajo, expusieron las actividades de taller y la reflexión escrita. Es importante señalar que los estudiantes no solo reforzaron los conocimientos de otras áreas del currículo, sino que aplicaron conocimientos del diseño artístico, situación que favoreció el desarrollo de sus sentidos, habilidades y destrezas.

- Creación de logotipos. En esta experiencia, cada estudiante se dio a la tarea de seleccionar una de las áreas curriculares de su agrado y posteriormente diseñó un "logo" que la identificara. Durante el proceso de elaboración se escucharon opiniones de parte de los compañeros en cuanto al diseño y proceso de ejecución. Esta actividad, además de lograr los objetivos que se refieren a la composición $\mathrm{y}$ al diseño que se estudiaron como parte del contenido del programa de artes plásticas, el estudiante se sintió con libertad para emitir su criterio en cuanto a otros tópicos de su agrado o desagrado. Finalmente, cada estudiante al exponer su trabajo dentro del aula, debió fundamentar su diseño haciendo referencia a la estructura organizativa del mismo (textura, color, énfasis, balance y equilibrio). La actividad permitió el desarrollo de la criticidad, la creatividad, así como el respeto a las ideas de las demás personas.

- Proyectos de servicio institucional: Como su nombre lo indica, la organización de este tipo de proyectos le permitió a los jóvenes identificar problemas o carencias de diversa índole en su institución. En este proyecto, tanto el problema como su solución lo expresaron por medio de dibujos y con el empleo del carboncillo y la utilización de tizas con leche, azúcar y laca. Lo importante en esta actividad es que se aplicaron conocimientos y aprendizajes con el propósito de ofrecer soluciones creativas y apropiadas al problema. La exposición de los trabajos se realizó en los corredores de la institución; aunque también se puede realizar en lugares estratégicos de la comunidad.

- Las artes plásticas y la expresión musical. Algunos jóvenes organizaron actividades de expresión artística de manera vinculada con los profesores que imparten enseñanza de la música. En esta oportunidad ambas poblaciones (docentes y estudiantes) planificaron de manera conjunta el mismo tema: "Las mascaradas en nuestro país". Para el desarrollo de esta experiencia, los estudiantes que recibieron lecciones de artes plásticas elaboraron máscaras con la aplicación de diversas técnicas y materiales: bolsas de papel, pinturas, papeles de colores, entre otros. Quienes imparten educación musical prepararon instrumentos elaborados por ellos y se dieron a la tarea de organizar una cimarrona. La experiencia fue interesante pues el tratamiento del mismo contenido desde expresiones de arte distintas, emocionó y entusiasmó al estudiantado.

\section{Consideraciones finales}

Las conclusiones que a continuación se señalan, surgen desde el momento en que las investigadoras nos acercamos a la práctica pedagógica que desarrollamos y culminan con la planificación de experiencias metodológicas en instituciones de educación secundaria de parte de los profesores de artes plásticas que participaron en 
la investigación. Cabe señalar que en todo momento de destacó el concepto de "integración” entre las artes plásticas y otras áreas del currículo escolar.

- De acuerdo con la literatura estudiada, tanto la formación académica idónea y la experiencia profesional, como las creencias, concepciones y estilos de pensamiento respecto a su función como docente, influyeron de manera positiva en la planificación de las actividades que organizaron como parte de su trabajo de aula.

- La organización de un currículo integrado favoreció en los estudiantes el establecimiento de conexiones entre las diferentes áreas del currículo escolar y las que corresponden al área artística.

- Las producciones que realizaron los estudiantes, llámense proyectos, actividades o talleres, reflejaron de su autor o autores sus facultades cognitivas, socio-afectivas, así como las habilidades y destrezas.

- Los docentes comprendieron que las experiencias de taller o de expresión artística no pueden ser consideradas como actividades puramente racionales o científicas, ni enteramente artísticas o sensitivas, como tampoco únicamente útiles, sensibles o tecnológicas. Al organizar actividades de aprendizaje desde un planteamiento integrador, o sea, al integrar diversas áreas del currículum escolar, encontramos contenidos racionales, artísticos, así como otros que estimulan la sensibilidad y la afectividad.

- La planificación de actividades pedagógicas desde una visión integradora, favoreció el desarrollo de una actitud crítica ante las diversas situaciones que se le pueden presentar dentro y fuera del contexto escolar.

- Los proyectos se organizaron alrededor de temas y planeamientos de integración curricular, en los que los objetivos, contenidos, procedimientos metodológicos, los materiales y las técnicas de expresión plásticas, así como los intereses, experiencias, conocimientos previos y la edad de los estudiantes, fueron factores fundamentales para el logro de trabajos de calidad.

- Las experiencias favorecieron en cada participante su "autoidentificación", de manera que siempre se sintieron orgullosos de sus trabajos y motivados a continuar aplicando este tipo de experiencias.

Deseo concluir indicando que los colegiales lograron desarrollar, desde una perspectiva lúdica, sus facultades creativas, su sensibilidad, sus gustos personales, así como el reconocimiento de su potencial artístico considerado fundamental para el logro de una personalidad plena.

\section{Notas}

1. Las experiencias de observación se realizaron en cursos que ofrecen las Escuelas de Formación Docente y de Filosofía, a saber: Filosofía Patrística, Seminario de Realidad Nacional "Hombre, Educación y Sociedad", Expresión Creadora, Expresión Musical en la Educación Inicial II, Fundamentos de Didáctica y Artes Aplicadas a la Educación.

2. Se utiliza el término deconstruir en un sentido técnico para referirse al proceso de deshacer y volver a construir, ya que dicho proceso sintetiza lo que se espera lograr con la población participante respecto de su práctica pedagógica.

\section{Referencias bibliográficas}

Alvarado, Ana y Murano, Gabriela. (1990). El taller de plástica en la escuela. Teoría y práctica. Argentina: Editorial Troquel.

Castro Bonilla Julieta. (2004, JulioDiciembre). La enseñanza de las 
artes plásticas en la Universidad de Costa Rica. Revista Electrónica "Actualidades Investigativas de la Educación”, 2 (4), Artículo 5. Extraído el 19/10/2007 de http://revista.inie. ucr.ac.cr

Castro Bonilla Julieta. (2006). Experiencias metodológicas construidas a partir de la expresión artística, del conocimiento, de las teorías implícitas y de los modelos de pensamiento del docente. Revista Educación, 1(30), 201-215.

Eisner, Elliot. (2004). El arte y la creación de la mente. El papel de las artes visuales en la transformación de la conciencia. Barcelona-España: Paidós Arte y Educación.

Fierro, C., Fortuol, B. y Rosas, L. (2002). Transformando la práctica docente. Una propuesta basada en la investigación-acción. Maestros y Enseñanza México: Paidós.

Flores O., Rafael. (1999). Evaluación Pedagógica y Cognición. Bogotá, Colombia: McGraw-Hill Interamericana.

Gimeno, Sacristán. (1993). Comprender y transformar la enseñanza, Madrid: Editorial Morata.

Klingler, Cynthia y otros. (1997). Psicología Cognitiva. Estrategias en la práctica docente. México: Editorial Mc Graw-Hill.

Marcelo G., Carlos. (1987). El Pensamiento del Profesor. Barcelona-España: Ediciones CEAC.
Pérez, U. Iris. (2000). Didáctica de la educación plástica. El taller de arte en la escuela. Buenos Aires, Argentina: Editorial Magisterio del Río de la Plata.

Rodrigo, María José y otros. (1993). Las teorías implícitas. Una aproximación al conocimiento cotidiano, Madrid: Editorial Aprendizaje Visor.

Romero O., Hernando. (1998). Metodología para formar docentes investigadores investigando. Pedagogía Constructivista de la Transformación Tomo 1l. Colombia: Ediciones Fundación CEINPE.

Rueda Beltrán y Díaz Barriga. (2000). Evaluación de la docencia. México: Paidós.

Universidad de Costa Rica. (1991). Plan de Profesorado y Bachillerato en la Enseñanza. Escuela de Formación Docente. s.e.

Universidad de Costa Rica. Consejo Universitario. (2000). Estatuto Orgánico. San José: Edición gráfica, Centro de Información y Servicios Técnicos, Unidad de Comunicación.

Vasco, Eloísa. (1995). Maestros, alumnos y saberes. Bogotá: Cooperativa Editorial Magisterio.

Venegas, Alicia. (2002). Las artes plásticas en la educación artística y estética infantil. México. D. F.: Ediciones Paidós Ibérica, S.A. 
